\title{
Fission Chambers Detector Unit Mockup Testing at Research
}

\section{Reactors}

\author{
V. P. Alferov, V. A. Fedorov, A. V. Kudryavtsev, E. S. Martazov, N. A. Selyaev, \\ ${ }^{1}$ A. V. Batyunin, ${ }^{1} \mathrm{Yu}$. A. Kashchuk and ${ }^{1}$ V. A. Vorobiev \\ National Research Nuclear University MEPhI, ${ }^{1}$ Institution "Project Center ITER"
}

The required range of neutron radiation intensity monitoring of the International Experimental Thermonuclear Reactor (ITER) is from $10^{14}$ to $10^{21} \mathrm{n} / \mathrm{s}$. One of the systems of neutron diagnostics, called the Divertor Neutron Flux Monitor (DNFM), contains as primary converters fission chambers (FCs) placed in the ITER Vacuum Vessel beneath the Divertor Dom. During the operation the FCs will be exposed to the intense neutron radiation and electromagnetic fields. The expected neutron flux at the location of the DNFM FC is $10^{6}$. $10^{13} \mathrm{n} \cdot \mathrm{cm}^{-2} \cdot \mathrm{s}^{-1}$.

A distinctive feature of the neutron diagnostic channel for ITER is the desired time resolution of $1 \mathrm{~ms}$ and a measuring error of $10 \%$. Such a requirement can be realized only at an FC signal registration rate of at least $1 \cdot 10^{5} \mathrm{~s}^{-1}$. Therefore, the pulse mode of processing the FC signals is very limited and it will probably be used only for calibration and diagnostics of the equipment. The working monitoring range of the channel with one $\mathrm{FC}$ will be less than 7 orders of magnitude. This explains the necessity of using several FCs with different sensitivity in the DNFM. The hardware of the signal processing equipment will be the same for all FCs.

The wide-range channel equipment circuitry development for DNFM recording signals is based on the experience of the MEPhI in the development, implementation and operation of wide-range neutron flux monitoring equipment for control and protection systems (CPS) for various types of nuclear reactors. An important factor in the design of such equipment is the use of the fluctuation (Campbell) mode of fission chamber operation and signal processing equipment. The use of the fluctuation mode made it possible to radically solve the problem of the neutron flux monitoring in CPS channels in the 10-order range using a single FC. The equipment operates in the pulse, fluctuation and current modes.

The work which related to the testing of the DNFM detector unit (DU) mockup by the influence of intensive fluxes of neutron and gamma radiation and evaluation of technical decisions of the neutron flux monitoring channel was performed with use of the research reactors. The fission chamber Signals Processing Unit (SPU) was used. This device based on microprocessors with wide application of high integration chips, including complex programmable logic devices (CPLD). Taking into account the requirements for ITER neutron diagnostics and the conditions for DU testing, the SPU circuitry was adjusted and the software of microprocessors and CPLDs was modified to control the temperature of the DU. In addition, a test complex including hardware and software for controlling equipment, processing and recording measurement data is assembled for a detailed research of the DU characteristics.

The test complex includes the remote thermo assembly and the control and signal processing equipment. Thermo assembly includes an electric heater $(\mathrm{EH})$ and a thermocouple (TC). Control and signal processing equipment consist of heater power supply (PS), DU signals processing unit (SPU), industrial personal computer (IPC), providing recording of signals and control of the SPU operation modes and automatic test unit (ATU) for recording the shape of pulses and the pulse height amplitude spectrum of fission chambers.

The DNFM detector unit mockup on the base of a fission chamber KNT 25 with a communication line was tested at the first stage of the tests. The mass of uranium in the chamber is equal to $1 \mathrm{~g}$ and the electrodes coating density is $1 \mathrm{mg} / \mathrm{cm}^{2}$. The communication line was made of a double coaxial cable with fiberglass insulation with length of more than $3 \mathrm{~m}$. The fission chamber with the communication line was placed in a sealed enclosure filled with an inert gases 
mixture with a pressure of up to $5 \cdot 10^{5} \mathrm{~Pa}$. At the second stage the mockup of a similar design based on the KNT-30 fission chamber with a uranium mass of $100 \mathrm{mg}$ was tested. For testing DU at temperatures up to $350{ }^{\circ} \mathrm{C}$ it was mounted in a thermo assembly with an electric heater and thermocouple.

The SPU provides receiving of the pulse-current signal from the DU and signals from the temperature sensors, amplification, digital processing and transmission in digital code of the measuring and diagnostic information to the IPC. Also the SPU controls the power supply of the electric heater EH by commands from the IPC.

The measurement methods and SPU software were developed for carrying out laboratory and reactor tests of the equipment. They provide:

- parameters setting, acquisition and archiving of information coming from the SPU;

- data processing and heater control of the thermal assembly with DU;

- real time display of data on all signals and parameters (controlled and calculated) on the screen in digital and graphical form;

- presentation, editing and printing of digital and graphical information of measurement results and processing.

The SPU provides DU fission chamber bias by a DC high-voltage power supply in the range from 0 to $500 \mathrm{~V}$ which controlled by commands from the IPC and provides the threshold voltage generation for the $\mathrm{FC}$ analog processing module.

The FC analog processing module consist of three measuring chains - pulse, fluctuation and current. The DU fission chamber signal enters to the differential input of the FC analog processing module which provides:

- amplification and preliminary conversion of FC pulses, fluctuation and average current;

- noise and current pulses discrimination caused by gamma radiation;

- FC current pulses transformation to standard form voltage pulses and calculation by

CPLD of the counting rate (pulse chain);

- logarithmic transformation and pre-filtering of the average and fluctuation FC currents.

The experiments to determine the DNFM detector unit mockup and the wide-range channel for monitoring the neutron flux characteristics were performed at the research reactors of the IRT MEPhI and VVR-c. The detector unit mockup was studied under DU temperature range from $20^{\circ} \mathrm{C}$ to $350^{\circ} \mathrm{C}$ with the neutron flux range from $10^{3}$ to $10^{12} \mathrm{n} \cdot \mathrm{cm}^{-2} \cdot \mathrm{s}^{-1}$.

The tests confirmed the ability to control the change in reactor power using the SPU fluctuation chains before and after the neutron fluence of $4.2 \cdot 10^{15} \mathrm{n} \cdot \mathrm{cm}^{-2}$ in the range of four decades with the FC background current up to $1 \cdot 10^{-4} \mathrm{~A}$. The measuring chains operating ranges in term of the equivalent count rate are determined as a result of processing the measurement data for pulse chain up to $1 \cdot 10^{6} \mathrm{~s}^{-1}$, for fluctuation chain from $4 \cdot 10^{5}$ to $4 \cdot 10^{9} \mathrm{~s}^{-1}$ and for current chain from $2 \cdot 10^{8}$ to $1 \cdot 10^{10} \mathrm{~s}^{-1}$.

The lower boundaries of the operating ranges for the fluctuation and current chains can be reduced several times by taking into account their background signals. Integral nonlinearity of all measuring chains in operating ranges does not exceed $\pm 2 \%$.

Satisfaction with speed requirements ( $1 \mathrm{~ms}$ measurement cycle) leads to a significant increase in statistical noise in measurements results for the pulse and fluctuation chains. We can estimate its contribution to the statistical error at different power levels and refine the measurement chains ranges boundaries using the ratio of the rms deviation $\left(\sigma_{\mathrm{F}}\right)$ to the average value of the signal (F) as a noise parameter. Therefore, the lower limit of the pulse chain must be increased to $4 \cdot 10^{5} \mathrm{~s}^{-1}$ and for the fluctuation chain up to $2 \cdot 10^{6} \mathrm{~s}^{-1}$ for a relative statistical noise of less than $5 \%$. 